\title{
Exciton-exciton interaction in transition metal dichalcogenide monolayers and van der Waals heterostructures
}

\author{
Daniel Erkensten $\odot,{ }^{1, *}$ Samuel Brem $\odot,{ }^{2}$ and Ermin Malic $\odot^{1,2}$ \\ ${ }^{1}$ Department of Physics, Chalmers University of Technology, 41296 Gothenburg, Sweden \\ ${ }^{2}$ Department of Physics, Philipps-Universität, 35037 Marburg, Germany
}

(Received 16 June 2020; accepted 13 January 2021; published 25 January 2021; corrected 28 January 2021)

\begin{abstract}
Due to a strong Coulomb interaction, excitons dominate the excitation kinetics in two-dimensional (2D) materials. While Coulomb scattering between electrons has been well studied, the interaction of excitons is more challenging and remains to be explored. As neutral composite bosons consisting of electrons and holes, excitons show nontrivial scattering dynamics. Here, we study exciton-exciton interaction in transition-metal dichalcogenides and related van der Waals heterostructures on microscopic footing. We demonstrate that the crucial criterion for efficient scattering is a large electron/hole mass asymmetry, giving rise to internal charge inhomogeneities of excitons and emphasizing their cobosonic substructure. Furthermore, both exchange and direct exciton-exciton interactions are boosted by enhanced exciton Bohr radii. We also predict an unexpected temperature dependence that is usually associated with phonon-driven scattering, and we reveal an orders of magnitude stronger interaction of interlayer excitons due to their permanent dipole moment. The developed approach can be generalized to arbitrary material systems and will help to study strongly correlated exciton systems, such as moire super lattices.
\end{abstract}

DOI: 10.1103/PhysRevB.103.045426

\section{INTRODUCTION}

The emergence of atomically thin two-dimensional (2D) materials, such as graphene and monolayer transition metal dichalcogenides (TMDs), has initiated a new research field offering a platform for the investigation of many-body correlations and quantum phenomena [1-4]. The strong Coulomb interaction in TMD monolayers gives rise to excitons, dominating the optical response, relaxation dynamics, and transport characteristics [5-8]. Excitons are neutral composite bosons (cobosons) consisting of Coulomb-bound electrons and holes. Due to a complex band structure exhibiting multiple valleys, there is a variety of different exciton species in TMDs including bright, momentum, and spin-dark intralayer excitons [9-11], as well as spatially separated interlayer excitons in van der Waals heterostructures [12-15].

Previous theoretical studies have addressed the fundamental many-body processes governing the phonon-driven exciton dynamics in monolayer TMDs [11,16-19] and van der Waals heterostructures [20-22]. In the considered weakexcitation regime, excitons were treated as noninteracting bosons. However, as the excitation density increases, the

\footnotetext{
*Corresponding author: daniel.erkensten@ chalmers.se
}

Published by the American Physical Society under the terms of the Creative Commons Attribution 4.0 International license. Further distribution of this work must maintain attribution to the author(s) and the published article's title, journal citation, and DOI. Funded by Bibsam. cobosonic nature of excitons comes to the surface and excitonexciton scattering becomes increasingly important [23-25]. While scattering between electrons has been treated extensively in literature [26,27], a microscopic treatment of the Coulomb interaction of excitons as neutral cobosonic quasiparticles has proven to be challenging [28-30]. The role of direct and exchange exciton-exciton interactions in TMDs has been previously discussed, but exclusively for bright excitons interacting in a monolayer [25]. In particular, exciton-exciton scattering, incorporating the remarkable intervalley and interlayer excitonic landscape in 2D materials, has so far remained unrevealed.

In this work, we investigate exciton-exciton scattering in TMD monolayers and van der Waals heterostructures based on a microscopic and quantum-mechanic approach. We evaluate the efficiency of the exciton-exciton interaction by calculating the excitation-induced dephasing (EID) of excitonic resonances in the incoherent limit. We resolve the underlying intravalley and intervalley as well as intralayer and interlayer exciton-exciton scattering channels, cf. Fig. 1. We reveal an intriguing temperature and screening dependence of EID and provide microscopic insights into the fundamental nature of scattering between intralayer and interlayer excitons. The latter exhibit a permanent out-of-plane dipole moment, and their interaction can be considered as an efficient dipoledipole coupling [Fig. 1(a)] resulting in an EID in the range of a few meV. In contrast, intralayer excitons do not have a permanent dipole moment and interact through higher-order electric moments induced by the internal charge inhomogeneity of excitons as cobosonic quasiparticles. Here, we show that the mass asymmetry between electrons and holes, as well as the overlap of Bohr radii, are the key quantities 
(a)

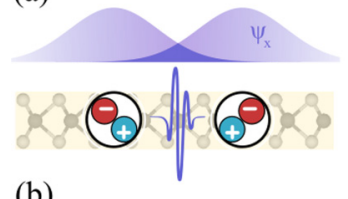

(b)

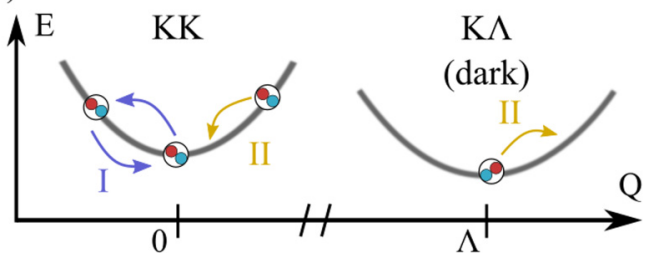

FIG. 1. (a) Schematic illustration of exciton-exciton scattering in a TMD monolayer (left) and a van der Waals heterostructure (right). While the intralayer interaction is determined by the wave function overlap, the interlayer coupling resembles a dipole-dipole interaction. (b) Exemplary scattering channels in monolayer $\mathrm{WSe}_{2}$ including intravalley (I, blue) and intervalley processes (II, orange).

determining the exciton-exciton scattering efficiency. The gained microscopic insights are applicable to a broader class of excitonic, multi-valley materials.

\section{THEORETICAL MODEL}

To investigate exciton-exciton scattering on a microscopic footing in 2D materials, we first define the many-particle Hamilton operator. Following the approach described in Ref. [30], we derive the excitonic Hamilton operator from the conventional electron-electron Hamiltonian by employing an identity operator expansion [31] and rewriting the electron and hole creation and annihilation operators $c^{(\dagger)}$ and $v^{(\dagger)}$ into excitonic operators $P^{(\dagger)}$. We obtain $H=H_{0}+H_{x-x}$, where the interaction-free part $H_{0}$ includes the excitonic energies $\epsilon_{Q}^{\alpha}=$ $E^{\alpha}+\frac{\hbar^{2}|\boldsymbol{Q}|^{2}}{2 M^{\xi}}, \boldsymbol{Q}$ being the center-of-mass momentum, $M^{\xi}$ the valley-dependent exciton mass, $E^{\alpha}$ the excitonic eigenenergies, and $\alpha=(n, \xi)$. The latter is a compound index including the excitonic state $n=1 s, 2 s \ldots$ in the valley $\xi=\left(\xi_{h}, \xi_{e}\right)=$ $\mathrm{KK}, \mathrm{KK}$ ', $\mathrm{K} \Lambda$, i.e., in this work we consider the hole to be localized around the $\mathrm{K}$ point, while the electron lies either around the $\mathrm{K}, \mathrm{K}^{\prime}$, or $\Lambda$ point (also often referred to as $Q$ point in literature [32]). Moreover, we focus on spin-allowed transitions [Fig. 2(a)]. In principle, exciton-exciton scattering including spin-dark states is also possible, but we expect this type of scattering to be qualitatively similar to the scattering with momentum-dark states that have been considered in this work.

The eigenenergies $E^{\alpha}$ and the associated excitonic wave functions $\varphi_{\alpha, \boldsymbol{q}}$ are obtained by solving the Wannier equation $[16,20]$, which is derived within the effective-mass approximation [33]. Thus, the Wannier equation provides reliable excitonic binding energies when the exciton is well localized in momentum space and the parabolic approximation holds [34]. In the case of monolayers, we treat the screened Coulomb potential entering the Wannier equation within the Keldysh approach [35-37] to account for the finite width of the TMD and screening effects. For charge carriers within a heterostructure, we solve the Poisson equation for

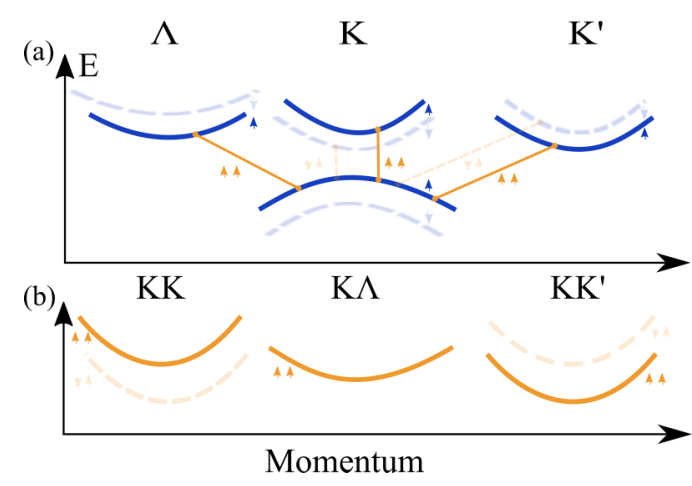

FIG. 2. (a) Schematic illustration of the electronic band structure of monolayer $\mathrm{WSe}_{2}$ in the vicinity of the high symmmetry $\mathrm{K}, \mathrm{K}^{\prime}$, and $\Lambda$ points. In this work, we focus on spin-allowed transitions (yellow lines). (b) Excitonic band structures displaying the energetic ordering of exciton states as a function of the center-of-mass momentum. Note that the momentum-dark KK' and $\mathrm{K} \Lambda$ states are energetically lower than the bright KK exciton.

two aligned homogeneous slabs, resulting in a generalized Keldysh screening [20]. The obtained excitonic eigenenergies can be found in Table I in the Supplementary Material [38] and are in good agreement both with DFT studies [39] and experimental observations [40]. In Fig. 2(b), the corresponding center-of-mass-dependent exciton dispersion $\epsilon_{Q}^{\alpha}$ is schematically illustrated for monolayer $\mathrm{WSe}_{2}$ showing the $n=1 s$ state of bright $\mathrm{KK}$ and momentum-dark $\mathrm{KK}^{\prime}, \mathrm{K} \Lambda$ excitons. Since the latter are energetically lowest states, we expect that exciton-exciton scattering involving both bright and dark excitons will be efficient even at room temperature.

The second part of the Hamiltonian of interest in this work is the exciton-exciton interaction $H_{x-x}$ given by

$$
H_{x-x}=\frac{1}{2} \sum \mathcal{W}_{\boldsymbol{q}}^{\alpha \beta \beta^{\prime} \alpha^{\prime}} P_{\alpha, \boldsymbol{Q}+\boldsymbol{q}}^{\dagger} P_{\beta, \boldsymbol{Q}^{\prime}-\boldsymbol{q}}^{\dagger} P_{\beta^{\prime}, \boldsymbol{Q}^{\prime}} P_{\alpha^{\prime}, \boldsymbol{Q}},
$$

with $\mathcal{W}_{\boldsymbol{q}}^{\alpha \beta \beta^{\prime} \alpha^{\prime}}=\left(\mathcal{V}_{\boldsymbol{q}}^{\alpha \beta \beta^{\prime} \alpha^{\prime}}+2 \mathcal{U}_{\boldsymbol{q}}^{\alpha \beta \beta^{\prime} \alpha^{\prime}}\right)$, and where summation over the excitonic indices $\alpha^{(\prime)}, \beta^{(\prime)}$, and momenta $\boldsymbol{Q}^{(\prime)}, \boldsymbol{q}$ is implied [30]. The matrix element $\mathcal{W}_{\boldsymbol{q}}^{\alpha \beta \beta^{\prime} \alpha^{\prime}}$ includes direct electron-electron, hole-hole, and electron-hole interactions $\left(\mathcal{V}_{\boldsymbol{q}}^{\alpha \beta \beta^{\prime} \alpha^{\prime}}\right)$ and exchange interactions $\left(\mathcal{U}_{\boldsymbol{q}}^{\alpha \beta \beta^{\prime} \alpha^{\prime}}\right)$. In this work, we focus on the most relevant valley conserving exciton-exciton scattering processes $\left(\alpha=\alpha^{\prime} \equiv \mu\right.$ and $\left.\beta=\beta^{\prime} \equiv v\right)$ involving a small momentum transfer in the energetically lowest $n=1 s$ state, and therefore intervalley interactions have been omitted in the exciton-exciton Hamiltonian (1). Figure 1(b) illustrates exemplary scattering processes involving only KK states (process I) and including different valleys (process II). The direct part of the monolayer excitonic Coulomb matrix element reads

$$
\left.\mathcal{V}_{\boldsymbol{q}}^{\mu \nu v \mu}\right|_{\text {mono }}=W_{\boldsymbol{q}} D_{\mu}(\boldsymbol{q}) D_{\nu}^{*}(\boldsymbol{q}),
$$

with the screened Coulomb potential $W_{\boldsymbol{q}}$ and $D_{\mu}(\boldsymbol{q})=$ $\left(F_{\mu}\left(\beta^{\mu} \boldsymbol{q}\right)-F_{\mu}^{*}\left(\alpha^{\mu} \boldsymbol{q}\right)\right)$ including the form factors $F_{\mu}(x \boldsymbol{q})=$ $\sum_{\boldsymbol{q}_{1}} \varphi_{\mu, \boldsymbol{q}_{1}+x \boldsymbol{q}}^{*} \varphi_{\mu, \boldsymbol{q}_{1}}$. The latter include the mass ratios $\alpha^{\mu}=$ $\frac{m_{e}^{\mu_{e}}}{m_{e}^{\mu_{e}}+m_{h}^{\mu_{h}}}, \beta^{\mu}=1-\alpha^{\mu}$, where $m_{e(h)}^{\mu_{e(h)}}$ is the electron (hole) mass in the valley $\mu_{e(h)}=\mathrm{K}, \Lambda, \mathrm{K}$. When considering scattering processes within a heterostructure, the form factors 

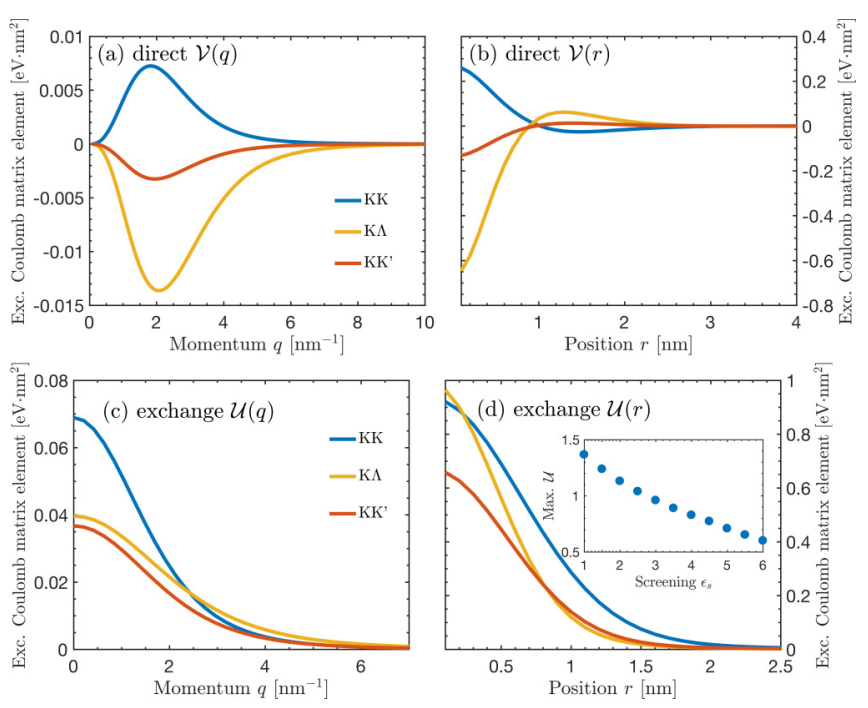

FIG. 3. Momentum and real space representations of the excitonic Coulomb matrix element in the $\mathrm{WSe}_{2}$ monolayer. We distinguish between direct interactions $\mathcal{V}$, illustrated in (a)-(b), and exchange interactions $\mathcal{U}$, shown in (c)-(d). We consider intravalley and intervalley exciton-exciton scattering [i.e., $\mu=\mathrm{KK}$ and $v=\mathrm{KK}$, $\mathrm{K} \Lambda$, KK', respectively, cf. also Fig. 1(b)] in $\mathrm{WSe}_{2}$. The inset in (d) displays the screening dependence of the exchange interaction element with $\mu=\nu=\mathrm{KK}$.

are weighted differently depending on whether the scattering occurs between interlayer excitons exclusively or between intralayer and interlayer excitons, cf. the Supplemental Material. The exchange contribution to the interaction reads

$$
\mathcal{U}_{\boldsymbol{q}}^{\mu \nu v \mu}=\frac{1}{2}\left(\delta_{\mu_{h}, v_{e}} \hat{\mathcal{U}}_{\boldsymbol{q}}^{\mu \nu}+\delta_{v_{h}, \mu_{e}} \hat{\mathcal{U}}_{-\boldsymbol{q}}^{\nu \mu}\right),
$$

with $\hat{\mathcal{U}}_{\boldsymbol{q}}^{\mu \nu}=\sum_{\boldsymbol{q}_{1}, \boldsymbol{q}_{2}} \tilde{W}_{\boldsymbol{q}_{2}-\boldsymbol{q}_{1}}^{\mu \nu} G_{\mu}\left(\boldsymbol{q}_{1}, \alpha^{\mu} \boldsymbol{q}\right) G_{v}\left(\boldsymbol{q}_{2}, \beta^{\nu} \boldsymbol{q}\right)$. Here we introduced $G_{\mu}\left(\boldsymbol{q}_{i}, x \boldsymbol{q}\right) \stackrel{\boldsymbol{q}_{2}}{=} \varphi_{\mu, \boldsymbol{q}_{i}}^{*} \varphi_{\mu, \boldsymbol{q}_{i}+x \boldsymbol{q}}$ with $i=1,2$ and $\tilde{W}_{q}^{\mu \nu}=W_{\boldsymbol{q}}\left(\boldsymbol{q} \cdot \boldsymbol{M}_{\mu_{h}}\right)\left(\boldsymbol{q} \cdot \boldsymbol{M}_{v_{e}}^{*}\right)$, where $\boldsymbol{M}_{\eta}$ is the optical matrix element describing the transition probability between the conduction and valence bands in the same valley $\eta=\mu_{h}, v_{e}$ [33,41]. We obtain $\boldsymbol{M}_{\eta}$ using $k \cdot p$ theory $[42,43]$ and deduce $\left|\boldsymbol{M}_{\eta} \cdot \mathbf{e}_{\sigma_{ \pm}}\right|=M_{\eta, \sigma_{ \pm}}=\frac{a_{0}}{\sqrt{2} E_{g}^{\eta \eta}} \eta^{\eta}\left(1 \pm \delta_{\eta, \mathrm{K}}\right)$ for left-handed $\left(\sigma_{+}\right)$ and right-handed $\left(\sigma_{-}\right)$circularly polarized light in the vicinity of $\eta=\mathrm{K}$. The lattice constant $a_{0}$, the band gap $E_{g}^{\eta \eta}$, and the overlap integrals $t^{\eta}=\left\langle u_{c, \eta}|\hat{\mathbf{p}}| u_{v, \eta}\right\rangle$ with the Bloch factors $u_{\lambda, \eta}$ are extracted from DFT calculations $[32,44]$. Note that the exchange interaction is only nonzero when the hole valley of one of the two scattering excitons is identical to the electron valley of the other exciton involved in the scattering process. Figure 3 illustrates the excitonic Coulomb matrix element in a hBN-encapsulated $\mathrm{WSe}_{2}$ monolayer considering different scattering channels with $\mu=\mathrm{KK}$ and $v=\mathrm{KK}, \mathrm{K} \Lambda, \mathrm{KK}^{\prime}$ in real and momentum space. We distinguish between direct $\mathcal{V}$ [Figs. 3(a) and 3(b)] and exchange contributions $\mathcal{U}$ [Figs. 3(c) and 3(d)]. We proceed by discussing these contributions separately, starting with the direct interaction. Since excitons are effectively neutral quasiparticles composed by electrons and holes, the resulting direct interaction potential in real space is reminiscent of the Lennard-Jones potential, cf. Fig. 3(b) [45]. We find that both repulsive or attractive exciton-exciton interactions occur for different exciton species and length scales. Our model predicts that the strength of the direct interaction is determined by the mass ratios of electrons and holes of the involved excitons. This can be seen by performing a Taylor expansion of Eq. (2) for small $q$ giving $\mathcal{V}_{q}^{\mu \nu \nu \mu} \approx$ $W_{q} r_{\mu, B}^{2} r_{\nu, B}^{2} q^{4} Q_{\mu} Q_{v}$ with $r_{\mu, B}^{2}=\left\langle\mu\left|r^{2}\right| \mu\right\rangle$, where $r_{\mu, B}$ is the excitonic Bohr radius. Furthermore, we have introduced the effective excitonic charge

$$
Q_{\mu}=\left(Q_{h} m_{h}^{\mu_{h}}+Q_{e} m_{e}^{\mu_{e}}\right) /\left(m_{h}^{\mu_{h}}+m_{e}^{\mu_{e}}\right),
$$

with $Q_{h / e}= \pm 1$ determining the sign of the interexcitonic potential and thus dictating the repulsive or attractive nature of the interaction. The latter can be interpreted as a force resulting from the internal charge inhomogeneity of the exciton. In particular, an exciton with a heavy hole will be positively charged in its center, surrounded by a negatively charged shell resulting from the orbiting electron. Considering the effective excitonic charges, the matrix element in the long-range limit is always positive for $\mu=v$, while it becomes negative if the interacting excitons have inverted mass ratios. For example, we find an attractive character at small distances for the KK$\mathrm{K} \Lambda$ and $\mathrm{KK}-\mathrm{KK}$ ' interaction in Fig. 3(b). While holes are heavier than electrons for KK excitons $\left(Q_{\mu}>0\right)$, the opposite is the case for $\mathrm{K} \Lambda$ and $\mathrm{KK}^{\prime}\left(Q_{v}<0\right)$. Moreover, having the hole at the $\mathrm{K}$ point and the electron at the $\Lambda$ point rather than at the $\mathrm{K}^{\prime}$ point increases the exciton-exciton interaction by a factor of 10 in momentum space, cf. Fig. 3(a). This is a direct consequence of a larger mass asymmetry, and consequently larger effective charge for $\mathrm{K} \Lambda$ excitons $\left(m_{h}^{\mathrm{K}}=\right.$ $0.36 m_{0}$ and $m_{e}^{\Lambda} \approx 0.6 m_{0}$, vs $m_{e}^{\mathrm{K}^{\prime}}=0.4 m_{0}$ [32]). Moreover, the direct interaction strongly depends on the excitonic Bohr radius (expected distance between electron and hole) as it scales with the quadropole moments of the excitons.

In contrast to the direct contribution, the exchange interaction $\mathcal{U}$ is nonvanishing at $\boldsymbol{q}=0$ and dominates in the considered momentum range. This behavior has already been established for interacting (bright) excitons in semiconductor quantum wells [46]. Moreover, the interaction is always repulsive and not highly sensitive to changes in electron and hole masses. In particular, the exchange interaction is only weakly reduced in momentum space when $\mathrm{KK}$ excitons are scattering with KK' excitons instead of K $\Lambda$ excitons, cf. Fig. 3(c). We also note that the exchange contribution can be estimated as $\mathcal{U}_{\boldsymbol{q}} \propto E_{B} r_{B}^{2}$ for $\boldsymbol{q} \ll 1$, where $E_{B}$ is the excitonic binding energy $[25,47,48]$.

Similar to the monolayer case, we perform an analysis of the interlayer excitonic Coulomb matrix element in a van der Waals heterostructure illustrated in Figure 4. Due to the spatial separation of electrons and holes forming interlayer excitons, we can neglect the exchange interaction, leaving us only with the direct contribution. In fact, as shown by a recent DFT study [49], the (squared) optical matrix element $|M|^{2}$ determining the strength of the exchange interaction is up to 200-300 times smaller for interlayer excitons in $\mathrm{MoSe}_{2}-\mathrm{WSe}_{2}$ compared to intralayer excitons.

In contrast to intralayer excitons, here the direct repulsion between electrons/holes is always stronger than the counteracting attraction of electrons and holes of different excitons. Therefore, the interaction between interlayer excitons can be understood as repulsion between two electric dipoles, cf. 

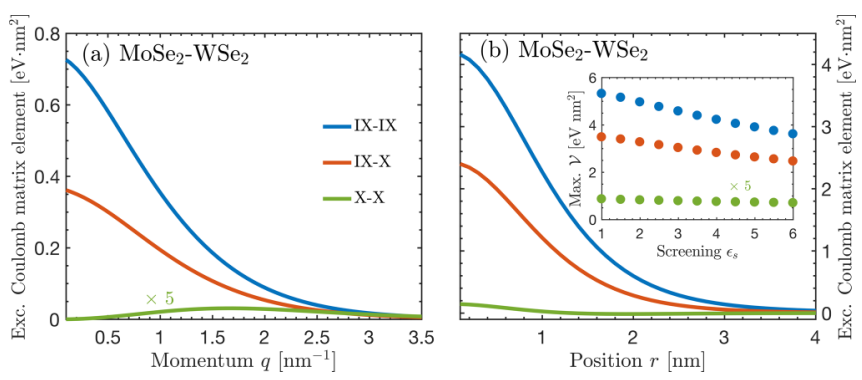

FIG. 4. Momentum and real space representations of the excitonic Coulomb matrix element in the $\mathrm{MoSe}_{2}-\mathrm{WSe}_{2}$ heterostructure. We consider scattering of $\mathrm{KK}$ interlayer excitons with other bright interlayer excitons (IX-IX) as well as with KK intralayer excitons (IX-X) and pure intralayer scattering between KK excitons (X-X). Note that the latter is strongly suppressed. The intralayer excitons we consider here reside in the $\mathrm{WSe}_{2}$ layer, and the interlayer excitons are composed from holes from the $\mathrm{MoSe}_{2}$ layer and electrons from the $\mathrm{WSe}_{2}$ layer. The inset in (b) illustrates the screening dependence of the matrix elements.

Fig. 1(a). As a consequence, the exciton-exciton interaction between interlayer excitons in the exemplary $\mathrm{MoSe}_{2}-\mathrm{WSe}_{2}$ heterostructure is up to two orders of magnitude larger than in the monolayer $\mathrm{WSe}_{2}$ case, cf. Figs. 3(c) and 3(d). Importantly, the direct interlayer and intralayer matrix elements also differ qualitatively. By expanding the direct matrix elements for small momenta, we find that $\left.\mathcal{V}_{q}^{\mu v v \mu}\right|_{\text {mono }} \propto W_{q} q^{4}$ vanishes for $q \rightarrow 0$, whereas the interlayer element remains nonzero, cf. Figs. 3(a) and 4(a), as well as the Supplemental Material [38], for details.

Based on the derived excitonic Coulomb matrix elements, we now calculate the dephasing of optical polarisations induced by exciton-exciton scattering that is referred to in literature as excitation-induced dephasing (EID) [50-52]. EID is a directly accessible phenomenon in experiments, and becomes manifest as a density-dependent broadening of excitonic transitions. In contrast to another recently published work [52], we evaluate the EID in the incoherent limit, i.e., in the long-time regime [33]. Note that we do not consider optical nonlinearities, but instead focus on the linear regime that is relevant for photoluminescence and transient absorption experiments.

We obtain microscopic access to the EID due to excitonexciton interactions by evaluating the Heisenberg equation of motion for the excitonic polarization $p_{\mu}=\left\langle P_{\mu, Q=0}\right\rangle$ in the light cone, i.e., $\boldsymbol{Q}=0$. When commuting the exciton-exciton Hamiltonian $H_{x-x}$ with the polarization, exciton-exciton correlations such as $S \propto\left\langle P^{\dagger} P^{\dagger} P\right\rangle$ need to be considered, which previously have been treated within the Hartree-Fock approximation [30]. Here, we go beyond this and evaluate the equations of motion in second-order Born-Markov approximation [33,50], cf. the Supplemental Material [38]. To linear order in exciton density, we obtain $\left.\dot{p}_{\mu}\right|_{H_{x-x}}=-\gamma_{Q=0}^{\mu} p_{\mu}$, introducing the excitation-induced dephasing

$$
\gamma_{\boldsymbol{Q}}^{\mu}(T)=\frac{\pi}{\hbar} \sum_{\nu \boldsymbol{Q}^{\prime} \boldsymbol{q}}\left|\mathcal{W}_{\boldsymbol{q}}^{\mu \nu v \mu}\right|^{2} N_{\boldsymbol{Q}^{\prime}}^{\nu}(T) \delta(\Delta \epsilon),
$$

with the delta function $\Delta \epsilon=\epsilon_{Q^{\prime}+q}^{\nu}-\epsilon_{Q^{\prime}}^{\nu}-\epsilon_{Q+q}^{\mu}+\epsilon_{Q}^{\mu}$ ensuring the energy conservation for the considered exciton-exciton scattering processes. The appearing temperature-dependent exciton occupation $N_{Q}^{\nu}(T)$ is estimated with an equilibrium Boltzmann distribution, parameterized by the total exciton density $n=\sum_{\nu Q} N_{Q}^{v}$.

In the following we focus on the EID of bright KK excitons and evaluate Eq. (5) for the state with $\boldsymbol{Q}=0$. In general, the expression has to be evaluated numerically, but in this particular case, where intravalley scattering $(\mu=v=\mathrm{KK})$ is dominant, the temperature and density dependence of EID can be addressed analytically yielding

$$
\left.\gamma_{0}^{\mathrm{KK}}(T)\right|_{\nu=\mathrm{KK}}=\frac{n}{\hbar} \sqrt{\frac{M^{\mathrm{KK}}}{8 \pi k_{B} T}} \int d q\left|\mathcal{W}_{q}^{\mathrm{KK}}\right|^{2} .
$$

We note a linear dependence of EID on exciton density $n$ as well as an explicit temperature dependence scaling with $T^{-\frac{1}{2}}$. Normally, temperature dependencies observed in linewidth experiments are attributed to the interaction with phonons [23]. However, our model predicts that the temperature dependent distribution of excitons in momentum space has a direct consequence on the amount of channels available for an energy and momentum conserving exciton-exciton scattering process. This results in an additional temperature-dependent broadening which can be experimentally separated from the phonon broadening by varying density and temperature.

\section{EXCITATION-INDUCED DEPHASING IN MONOLAYERS}

We exploit Eqs. (2) and (5) to determine the excitationinduced dephasing of bright $\mathrm{KK}$ excitons in a $\mathrm{hBN}$ encapsulated $\mathrm{WSe}_{2}$ monolayer. The choice of substrate is motivated by the fact that Auger processes [53-55], not captured by our theoretical model, are shown to be suppressed in hBN-encapsulated monolayers [56]. Here, we focus on the dominant intravalley scattering channels within the $K$, $\Lambda$, and K' valley, cf. Figs. 1(b) and 2(b). We illustrate the temperature and density dependence of the EID for the $\mathrm{WSe}_{2}$ monolayer in Fig. 5. As confirmed by earlier experiments $[23,57]$ and expected from our theory, the EID increases linearly with exciton density $n$, i.e., $\gamma^{\mathrm{KK}}=\gamma_{x-x} n$ with the slope $\gamma_{x-x}$. However, as demonstrated in Fig. 5(b), the slope of the EID is highly temperature dependent with $\gamma_{x-x}=2.9$. $10^{-10}\left(4.9 \cdot 10^{-10}\right) \mu \mathrm{eVcm}^{2}$ for $T=300(50 \mathrm{~K})$. This behavior is governed by the temperature dependence of the exciton distributions of the bright $\mathrm{KK}$ and momentum-dark $\mathrm{K} \Lambda$ and KK' states [Fig. 5(c)]. For low temperatures $(T<30 \mathrm{~K})$, KK' excitons determine the EID, since most excitons reside in this energetically lowest state, see Fig. 2(b) and Table I in the Supplemental Material [38] for the energetic hierarchy of excitonic states in $\mathrm{WSe}_{2}$. At elevated temperatures the EID is dominated by $\mathrm{K} \Lambda$ excitons reflecting their highest occupation, see Fig. 5(c). Hence, we have addressed the importance of dark excitonic states on the EID. Note that the intervalley scattering channels are expected to be most efficient for tungsten-based TMDs. In molybdenum-based TMDs, in particular $\mathrm{MoSe}_{2}$, where the KK state is the energetically lowest state [10], bright excitons are expected to dominate the EID 

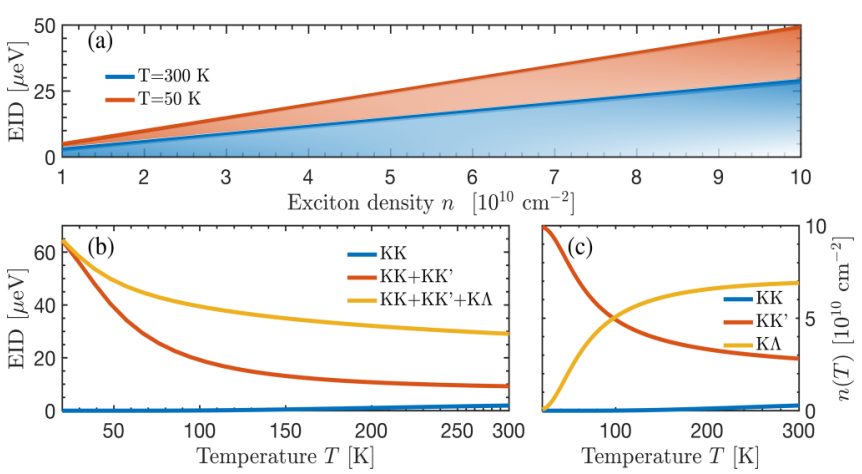

FIG. 5. Temperature and density dependence of excitationinduced dephasing in monolayer WSe $\mathrm{W}_{2}$. (a) EID as a function of exciton density $n$ for two different temperatures. (b) EID as a function of temperature, showing single scattering contributions including intravalley scattering $(\mathrm{KK})$ as well as intervalley scattering $\left(\mathrm{K} \Lambda, \mathrm{KK}^{\prime}\right)$ at fixed exciton density $n=10^{11} \mathrm{~cm}^{-2}$. (c) Temperaturedependent density $n(T)$ of KK, K $\Lambda$ and KK' excitons.

at all temperatures. Note that the quantitatively larger values of EID experimentally observed for the $\mathrm{WSe}_{2}$ monolayer on a sapphire substrate [23] have been obtained from coherent nonlinear spectroscopy experiments, where incoherent contributions studied in this work cannot be accessed.

\section{EXCITATION-INDUCED DEPHASING IN HETEROSTRUCTURES}

Now, we investigate the impact of excitation-induced dephasing on a $\mathrm{hBN}$-encapsulated $\mathrm{MoSe}_{2}-\mathrm{WSe}_{2}$ heterostructure. Here, both the EID of the intralayer (X) as well as of the spatially separated interlayer (IX) excitons within the heterostructure are considered. Note that we consider intralayer excitons in the $\mathrm{WSe}_{2}$ layer. According to a recent DFT study [58], KK interlayer excitons reside in the energetically lowest state in the $\mathrm{MoSe}_{2}-\mathrm{WSe}_{2}$ heterostructure. However, intervalley interlayer excitons, in particular the K $\Lambda$ state, can be found at a similar energy scale [59], and might also have an impact on the EID. However, treating the strong hybridisation at the $\Lambda$-point $[58,60]$ is beyond the scope of this work, and thus these states have not been considered here. Qualitatively, the analysis of the density dependence of the intralayer and interlayer EID is similar to the monolayer case, cf. Fig. 6(a). We find a linear density dependence, however with drastically higher slope values of $\gamma_{x-x}^{\mathrm{IX}}=1.2$. $10^{-11} \mathrm{meVcm}^{2}$ and $\gamma_{x-x}^{\mathrm{X}}=2.7 \cdot 10^{-12} \mathrm{meVcm}^{2}$. Due to the stronger exciton-exciton interaction for interlayer excitons exhibiting a permanent dipole moment (Fig. 3), the EID is two orders of magnitude larger compared to the $\mathrm{WSe}_{2}$ monolayer. Furthermore, due to the type-II band alignment in the $\mathrm{MoSe}_{2}-\mathrm{WSe}_{2}$ heterostructure and the large interlayer energy offset $\Delta E=315 \mathrm{meV}$ between the layers [20], the interlayer exciton state is rendered by far the lowest energetic state in agreement with [58]. Note that the chosen energy offset $\Delta E=315 \mathrm{meV}$ corresponds to an AA-stacked heterostructure, but the same insights on exciton-exciton scattering are expected also for other types of stacking (AA, AA', or $\mathrm{AB}$ ) provided a similar band offset $[59,61]$.
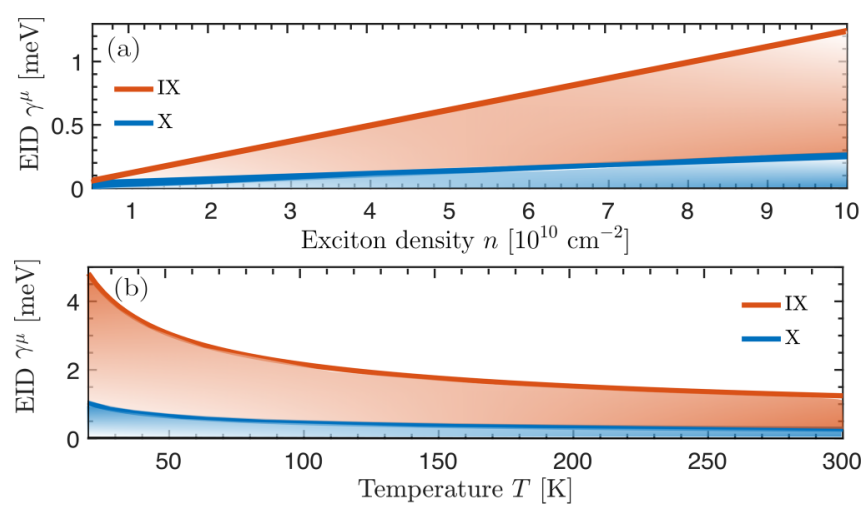

FIG. 6. Excitation-induced dephasing of interlayer (IX) and intralayer $\mathrm{KK}$ excitons (X) in the $\mathrm{MoSe}_{2}-\mathrm{WSe}_{2}$ heterostructure. (a) Density dependence of intralayer and interlayer EID at room temperature. (b) Temperature-dependent EID at fixed density $n=$ $10^{11} \mathrm{~cm}^{-2}$ revealing $\gamma^{\mathrm{I} / \mathrm{IX}}(T) \propto T^{-\frac{1}{2}}$.

Consequently, exclusively interlayer excitons contribute to the EID at all temperatures. In this particular case, the analytical formula in Eq. (6) can be used to evaluate the EID, predicting a $T^{-\frac{1}{2}}$ temperature dependence for interlayer excitons, as observed in Fig. 6(b).

Finally, we investigate how the choice of substrate affects the EID. In Fig. 7 the EID is shown as a function of the background dielectric screening $\epsilon_{s}$ for two different temperatures. We find a similar behavior for $T=50 \mathrm{~K}$ and $T=300 \mathrm{~K}$, namely that the EID in both the monolayer and the heterostructure displays a surprisingly moderate variation due to screening despite the strong screening dependence of the Coulomb potential itself. This reflects the moderate decrease of the (direct) excitonic Coulomb matrix elements with screening, as illustrated by the insets in Figs. 3(d) and 4(b). The background is that a larger screening also gives rise to an enhanced Bohr radius, which increases the form factors $F_{\mu}$ and $G_{\mu}$ appearing in the excitonic Coulomb matrix elements, cf. Eqs. (2) and (3), respectively. Hence, there is a competition between a decreased Coulomb potential $W_{q}$

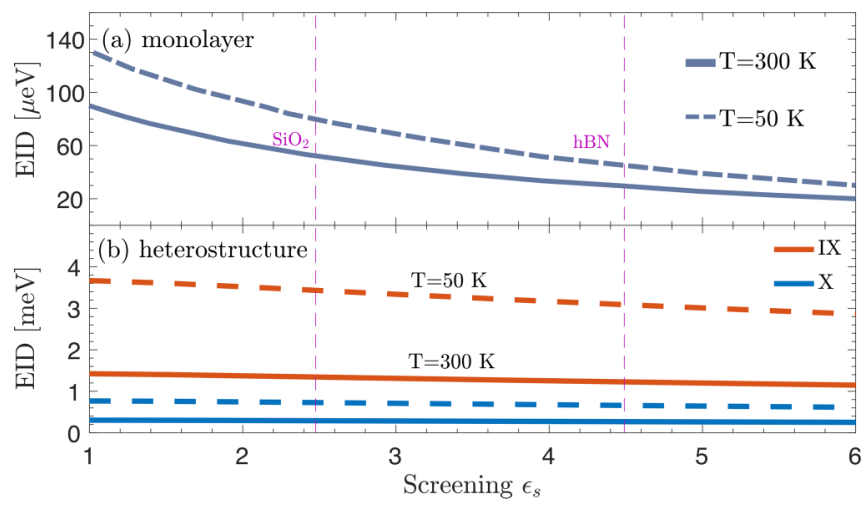

FIG. 7. Screening dependence of excitation-induced dephasing for the (a) $\mathrm{WSe}_{2}$ monolayer and the (b) $\mathrm{MoSe}_{2}-\mathrm{WSe}_{2}$ heterostructure for $T=50 \mathrm{~K}$ and $T=300 \mathrm{~K}$ and at the fixed exciton density $n=$ $10^{11} \mathrm{~cm}^{-2}$. Two of the most common substrates, $\mathrm{hBN}$ and $\mathrm{SiO}_{2}$, have been indicated as vertical dashed lines. 
and an increased Bohr radius resulting in a weak overall screening dependence. Still, we observe a stronger screening dependence in the monolayer case compared to heterostructures. This can be traced back to the exchange interaction that is dominating for monolayers and is more sensitive to screening than the direct contribution, which is crucial for heterostructures. In particular, the direct interaction displays a stronger dependence on the excitonic Bohr radius in the limit $\boldsymbol{q} \ll 1\left(\mathcal{V} \propto r_{B}^{4}\right)$ compared to the exchange interaction $\left(\mathcal{U} \propto E_{B} r_{B}^{2}\right)$, and hence the compensation between decreased Coulomb potential and increased Bohr radius is weakened when the exchange interaction is dominant.

\section{CONCLUSION}

We have presented a microscopic and quantum-mechanic approach on exciton-exciton scattering in 2D materials and related van der Waals heterostructures. To evaluate the scattering efficiency, we calculate the excitation-induced dephasing (EID) taking into account intravalley and intervalley as well as intralayer and interlayer exciton-exciton scattering chan- nels. We predict an intriguing temperature and screening dependence, and explain this by shedding light into the fundamental nature of exciton-exciton scattering. Spatially separated interlayer excitons in heterostructures exhibit a permanent dipole moment and their interaction can be considered as an efficient dipole-dipole coupling resulting in an EID of a few meV. In contrast, intralayer excitons are neutral cobosonic quasiparticles. The exciton-exciton scattering efficiency is boosted by a large electron/hole mass asymmetry and Bohr radii overlap of the excitons. The gained insights can guide future experimental studies on the impact of exciton-exciton scattering on optical properties of multivalley 2D materials.

\section{ACKNOWLEDGMENTS}

We thank Raul Perea-Causin (Chalmers) and Florian Katsch (TU Berlin) for fruitful discussions. We acknowledge funding from the European Union's Horizon 2020 research and innovation program under grant agreement No. 881603 (Graphene Flagship) as well as from the Swedish Research Council (VR, project number 2018-00734).
[1] K. S. Novoselov, D. V. Andreeva, W. Ren, and G. Shan, Graphene and other two-dimensional materials, Front. Phys. 14, 13301 (2019).

[2] Y. Cao, V. Fatemi, S. Fang, K. Watanabe, T. Taniguchi, E. Kaxiras, and P. Jarillo-Herrero, Unconventional superconductivity in magic-angle graphene superlattices, Nature 556, 43 (2018).

[3] F. Wu, T. Lovorn, E. Tutuc, and A. H. MacDonald, Hubbard Model Physics in Transition Metal Dichalcogenide Moiré Bands, Phys. Rev. Lett. 121, 026402 (2018).

[4] H. Yu, G.-B. Liu, J. Tang, X. Xu, and W. Yao, Moiré excitons: From programmable quantum emitter arrays to spin-orbitcoupled artificial lattices, Sci. Adv. 3, e1701696 (2017).

[5] G. Wang, A. Chernikov, M. M. Glazov, T. F. Heinz, X. Marie, T. Amand, and B. Urbaszek, Colloquium: Excitons in atomically thin transition metal dichalcogenides, Rev. Mod. Phys. 90, 021001 (2018).

[6] T. Mueller and E. Malic, Exciton physics and device application of two-dimensional transition metal dichalcogenide semiconductors, npj 2D Mater. App. 2, 29 (2018).

[7] H. Yu, X. Cui, X. Xu, and W. Yao, Valley excitons in twodimensional semiconductors, Natl. Sci. Rev. 2, 57 (2015).

[8] R. Perea-Causin, S. Brem, R. Rosati, R. Jago, M. Kulig, J. D. Ziegler, J. Zipfel, A. Chernikov, and E. Malic, Exciton propagation and halo formation in two-dimensional materials, Nano Lett. 19, 7317 (2019).

[9] T. Deilmann and K. S. Thygesen, Dark excitations in monolayer transition metal dichalcogenides, Phys. Rev. B 96, 201113(R) (2017)

[10] E. Malic, M. Selig, M. Feierabend, S. Brem, D. Christiansen, F. Wendler, A. Knorr, and G. Berghäuser, Dark excitons in transition metal dichalcogenides, Phys. Rev. Materials 2, 014002 (2018).

[11] M. Selig, G. Berghäuser, M. Richter, R. Bratschitsch, A. Knorr, and E. Malic, Dark and bright exciton formation, thermalization, and photoluminescence in monolayer transition metal dichalcogenides, 2D Materials 5, 035017 (2018)

[12] P. Rivera, J. R. Schaibley, A. M. Jones, J. S. Ross, S. Wu, G. Aivazian, P. Klement, K. Seyler, G. Clark, N. J. Ghimire, J. Yan, D. G. Mandrus, W. Yao, and X. Xu, Observation of long-lived interlayer excitons in monolayer mose2-wse2 heterostructures, Nat. Commun. 6, 6242 (2015).

[13] M. M. Fogler, L. V. Butov, and K. S. Novoselov, Hightemperature superfluidity with indirect excitons in van der waals heterostructures, Nat. Commun. 5, 4555 (2014).

[14] S. Latini, K. T. Winther, T. Olsen, and K. S. Thygesen, Interlayer excitons and band alignment in mos2/hbn/wse2 van der waals heterostructures, Nano Letters, Nano Lett. 17, 938 (2017).

[15] A. Surrente, Ł. Kłopotowski, N. Zhang, M. Baranowski, A. A. Mitioglu, M. V. Ballottin, P. C. M. Christianen, D. Dumcenco, Y.-C. Kung, D. K. Maude, A. Kis, and P. Plochocka, Intervalley scattering of interlayer excitons in a mos $2 / \operatorname{mose} 2 / \operatorname{mos} 2$ heterostructure in high magnetic field, Nano Lett. 18, 3994 (2018).

[16] S. Brem, M. Selig, G. Berghaeuser, and E. Malic, Exciton relaxation cascade in two-dimensional transition metal dichalcogenides, Sci. Rep. 8, 8238 (2018).

[17] M. Selig, G. Berghäuser, A. Raja, P. Nagler, C. Schüller, T. F. Heinz, T. Korn, A. Chernikov, E. Malic, and A. Knorr, Excitonic linewidth and coherence lifetime in monolayer transition metal dichalcogenides, Nat. Commun. 7, 13279 (2016).

[18] S. Brem, A. Ekman, D. Christiansen, F. Katsch, M. Selig, C. Robert, X. Marie, B. Urbaszek, A. Knorr, and E. Malic, Phonon-assisted photoluminescence from indirect excitons in monolayers of transition-metal dichalcogenides, Nano Lett. 20 , 2849 (2020).

[19] Z. Nie, Y. Shi, S. Qin, Y. Wang, H. Jiang, Q. Zheng, Y. Cui, Y. Meng, F. Song, X. Wang, I. C. E. Turcu, X. Wang, Y. Xu, Y. Shi, J. Zhao, R. Zhang, and F. Wang, Tailoring exciton dynamics of monolayer transition metal dichalcogenides by interfacial electron-phonon coupling, Commun. Phys. 2, 103 (2019). 
[20] S. Ovesen, S. Brem, C. Linderälv, M. Kuisma, T. Korn, P. Erhart, M. Selig, and E. Malic, Interlayer exciton dynamics in van der waals heterostructures, Commun. Phys. 2, 23 (2019).

[21] P. Merkl, F. Mooshammer, P. Steinleitner, A. Girnghuber, K. Q. Lin, P. Nagler, J. Holler, C. Schüller, J. M. Lupton, T. Korn, S. Ovesen, S. Brem, E. Malic, and R. Huber, Ultrafast transition between exciton phases in van der waals heterostructures, Nat. Mater. 18, 691 (2019).

[22] M.-L. Lin, Y. Zhou, J.-B. Wu, X. Cong, X.-L. Liu, J. Zhang, H. Li, W. Yao, and P.-H. Tan, Cross-dimensional electron-phonon coupling in van der waals heterostructures, Nat. Commun. 10, 2419 (2019).

[23] G. Moody, C. Kavir Dass, K. Hao, C.-H. Chen, L.-J. Li, A. Singh, K. Tran, G. Clark, X. Xu, G. Berghäuser, E. Malic, A. Knorr, and $\mathrm{X}$. Li, Intrinsic homogeneous linewidth and broadening mechanisms of excitons in monolayer transition metal dichalcogenides, Nat. Commun. 6, 8315 (2015).

[24] H. D. Sun, T. Makino, N. T. Tuan, Y. Segawa, Z. K. Tang, G. K. L. Wong, M. Kawasaki, A. Ohtomo, K. Tamura, and H. Koinuma, Stimulated emission induced by exciton-exciton scattering in zno/znmgo multiquantum wells up to room temperature, Appl. Phys. Lett. 77, 4250 (2000).

[25] V. Shahnazaryan, I. Iorsh, I. A. Shelykh, and O. Kyriienko, Exciton-exciton interaction in transition-metal dichalcogenide monolayers, Phys. Rev. B 96, 115409 (2017).

[26] A. Knorr and E. Malic, Graphene and Carbon Nanotubes (Wiley-VCH, Hoboken, 2013).

[27] T. R. Nielsen, P. Gartner, and F. Jahnke, Many-body theory of carrier capture and relaxation in semiconductor quantum-dot lasers, Phys. Rev. B 69, 235314 (2004).

[28] M. Combescot, O. Betbeder-Matibet, and R. Combescot, Exciton-exciton scattering: Composite boson versus elementary boson, Phys. Rev. B 75, 174305 (2007).

[29] M. Combescot, O. Betbeder-Matibet, and F. Dubin, The manybody physics of composite bosons, Phys. Rep. 463, 215 (2008).

[30] F. Katsch, M. Selig, A. Carmele, and A. Knorr, Theory of exciton-exciton interactions in monolayer transition metal dichalcogenides, Phys. Status Solidi (b) 255, 1800185 (2018).

[31] A. L. Ivanov and H. Haug, Self-consistent theory of the biexciton optical nonlinearity, Phys. Rev. B 48, 1490 (1993).

[32] A. Kormányos, G. Burkard, M. Gmitra, J. Fabian, V. Zólyomi, N. D Drummond, and V. Fal'ko, kp theory for two-dimensional transition metal dichalcogenide semiconductors, 2D Materials 2, 022001 (2015).

[33] M. Kira and S. W. Koch, Semiconductor Quantum Optics (Cambridge University Press, Cambridge, 2011).

[34] K. Zollner, P. E. Faria Junior, and J. Fabian, Giant proximity exchange and valley splitting in transition metal dichalcogenide/ $h \mathrm{BN} /(\mathrm{co}, \mathrm{ni})$ heterostructures, Phys. Rev. B 101, 085112 (2020).

[35] N. S. Rytova, The screened potential of a point charge in a thin film, Proc. MSU, Phys. Astron. 3, 30 (1967).

[36] L. V. Keldysh, Coulomb interaction in thin semiconductor and semimetal films, Sov. Phys. JETP 29, 658 (1979).

[37] P. Cudazzo, I. V. Tokatly, and A. Rubio, Dielectric screening in two-dimensional insulators: Implications for excitonic and impurity states in graphane, Phys. Rev. B 84, 085406 (2011).

[38] See Supplemental Material at http://link.aps.org/supplemental/ 10.1103/PhysRevB.103.045426 for additional information on excitonic binding energies, excitonic Coulomb matrix elements, excitation-induced dephasing, and input parameters-the latter being taken from references [32,62,63].

[39] T. Deilmann and K. S. Thygesen, Finite-momentum exciton landscape in mono- and bilayer transition metal dichalcogenides, 2D Materials 6, 035003 (2019).

[40] M. Goryca, J. Li, A. V. Stier, T. Taniguchi, K. Watanabe, E. Courtade, S. Shree, C. Robert, B. Urbaszek, X. Marie, and S. A. Crooker, Revealing exciton masses and dielectric properties of monolayer semiconductors with high magnetic fields, Nat. Commun. 10, 4172 (2019).

[41] H. Yu, G.-B. Liu, P. Gong, X. Xu, and W. Yao, Dirac cones and dirac saddle points of bright excitons in monolayer transition metal dichalcogenides, Nat. Commun. 5, 3876 (2014).

[42] J. M. Luttinger and W. Kohn, Motion of electrons and holes in perturbed periodic fields, Phys. Rev. 97, 869 (1955).

[43] E. O. Kane, Band structure of indium antimonide, J. Phys. Chem. Solids 1, 249 (1957).

[44] D. Xiao, G.-B. Liu, W. Feng, X. Xu, and W. Yao, Coupled Spin and Valley Physics in Monolayers of $\operatorname{mos}_{2}$ and Other Group-Vi Dichalcogenides, Phys. Rev. Lett. 108, 196802 (2012).

[45] E. J. Sie, A. Steinhoff, C. Gies, C. H. Lui, Q. Ma, M. Rösner, G. Schönhoff, F. Jahnke, T. O. Wehling, Y. H. Lee, J. Kong, P. Jarillo-Herrero, and N. Gedik, Observation of exciton redshiftblueshift crossover in monolayer ws2, Nano Lett. 17, 4210 (2017).

[46] C. Ciuti, V. Savona, C. Piermarocchi, A. Quattropani, and P. Schwendimann, Role of the exchange of carriers in elastic exciton-exciton scattering in quantum wells, Phys. Rev. B 58, 7926 (1998).

[47] M. M. Glazov, H. Ouerdane, L. Pilozzi, G. Malpuech, A. V. Kavokin, and A. D'Andrea, Polariton-polariton scattering in microcavities: A microscopic theory, Phys. Rev. B 80, 155306 (2009).

[48] F. Tassone and Y. Yamamoto, Exciton-exciton scattering dynamics in a semiconductor microcavity and stimulated scattering into polaritons, Phys. Rev. B 59, 10830 (1999).

[49] T. Woźniak, P. E. Faria Junior, G. Seifert, A. Chaves, and J. Kunstmann, Exciton $g$ factors of van der waals heterostructures from first-principles calculations, Phys. Rev. B 101, 235408 (2020).

[50] H. Haug and S. W. Koch, Quantum Theory of the Optical and Electronic Properties of Semiconductors, 5th ed. (World Scientific, Singapore, 2009).

[51] A. R. S. Kandada, H. Li, F. Thouin, E. R. Bittner, and C. Silva, Stochastic scattering theory for excitation induced dephasing: Time-dependent nonlinear coherent exciton lineshapes, J. Chem. Phys. 153, 164706 (2020).

[52] F. Katsch, M. Selig, and A. Knorr, Exciton-Scattering-Induced Dephasing in Two-Dimensional Semiconductors, Phys. Rev. Lett. 124, 257402 (2020).

[53] D. Sun, Y. Rao, G. A. Reider, G. Chen, Y. You, L. Brézin, A. R. Harutyunyan, and T. F. Heinz, Observation of rapid excitonexciton annihilation in monolayer molybdenum disulfide, Nano Lett. 14, 5625 (2014).

[54] B. Han, C. Robert, E. Courtade, M. Manca, S. Shree, T. Amand, P. Renucci, T. Taniguchi, K. Watanabe, X. Marie, L. E. Golub, M. M. Glazov, and B. Urbaszek, Exciton States in Monolayer Mose 2 and Mote 2 Probed by Upconversion Spectroscopy, Phys. Rev. X 8, 031073 (2018). 
[55] J. Binder, J. Howarth, F. Withers, M. R. Molas, T. Taniguchi, K. Watanabe, C. Faugeras, A. Wysmolek, M. Danovich, V. I. Fal'ko, A. K. Geim, K. S. Novoselov, M. Potemski, and A. Kozikov, Upconverted electroluminescence via auger scattering of interlayer excitons in van der waals heterostructures, Nat. Commun. 10, 2335 (2019).

[56] J. Zipfel, M. Kulig, R. Perea-Causín, S. Brem, J. D. Ziegler, R. Rosati, T. Taniguchi, K. Watanabe, M. M. Glazov, E. Malic, and A. Chernikov, Exciton diffusion in monolayer semiconductors with suppressed disorder, Phys. Rev. B 101, 115430 (2020).

[57] P. Merkl, F. Mooshammer, S. Brem, A. Girnghuber, K.-Q. Lin, L. Weigl, M. Liebich, C.-K. Yong, R. Gillen, J. Maultzsch, J. M. Lupton, E. Malic, and R. Huber, Twist-tailoring coulomb correlations in van der waals homobilayers, Nat. Commun. 11, 2167 (2020).

[58] X. Lu, X. Li, and L. Yang, Modulated interlayer exciton properties in a two-dimensional moiré crystal, Phys. Rev. B 100, 155416 (2019).
[59] R. Gillen and J. Maultzsch, Interlayer excitons in mose $_{2} / \mathrm{wse}_{2}$ heterostructures from first principles, Phys. Rev. B 97, 165306 (2018).

[60] S. Brem, K.-Q. Lin, R. Gillen, J. M. Bauer, J. Maultzsch, J. M. Lupton, and E. Malic, Hybridized intervalley moiré excitons and flat bands in twisted wse2 bilayers, Nanoscale 12, 11088 (2020).

[61] E. Torun, H. P. C. Miranda, A. Molina-Sánchez, and L. Wirtz, Interlayer and intralayer excitons in $\operatorname{mos}_{2} / \mathrm{ws}_{2}$ and $\mathrm{mose}_{2} / \mathrm{wse}_{2}$ heterobilayers, Phys. Rev. B 97, 245427 (2018).

[62] R. Geick, C. H. Perry, and G. Rupprecht, Normal modes in hexagonal boron nitride, Phys. Rev. 146, 543 (1966).

[63] A. Laturia, M. L. Van de Put, and W. G. Vandenberghe, Dielectric properties of hexagonal boron nitride and transition metal dichalcogenides: from monolayer to bulk, npj 2D Mater. App. 2, 6 (2018).

Correction: The fourth sentence of the abstract contained an error in wording and has been replaced. 